\title{
Generalized Semi Exponential Type Estimator under Systematic Sampling
}

\author{
Muhammad Nouman Qureshi ${ }^{1,3}$, Sadia Khalii ${ }^{2}$ and Muhammad Hanif ${ }^{3}$ \\ ${ }^{1}$ School of Statistics, University of Minnesota, Minneapolis-55455, USA \\ ${ }^{2}$ Department of Statistics, Lahore College for Women University, Lahore-54000, PAKISTAN \\ ${ }^{3}$ Department of Statistics, National College of Business Administration \& Economics, \\ Lahore-54660, PAKISTAN \\ nqureshi633@gmail.com sadia_khalil@hotmail.com drmianhanif@gmail.com
}

\begin{abstract}
In sample surveys, collection of auxiliary information together with the main variable of interest is very important to increase the efficiency of the estimators of population parameters of interest. Regression and ratio estimation are very popular and are widely used methods that benefit from the use of auxiliary information for the estimation of population parameters like mean, total, variance, proportion etc. A generalized semi-exponential type estimator is proposed in this paper using two auxiliary variables under the framework of systematic sampling. The expressions of approximate bias and mean square error of the proposed estimator have been derived. Algebraic conditions have been obtained under which the proposed estimator is more efficient than the competing estimators considered here. An empirical study has been carried out to show the improvement in efficiency of the proposed estimator as compared to the existing estimators.
\end{abstract}

Keywords: Auxiliary information, Generalized Semi-Exponential type estimator, Systematic Sampling, Mean Square Error, Percentage Relative Efficiency.

Copyright (C) 2018, the Authors. Published by Atlantis Press.

This is an open access article under the CC BY-NC license (http://creativecommons.org/licenses/by-nc/4.0/). 


\section{Introduction}

In survey sampling, no single estimation procedure will always work the best. Various sampling designs are available for different populations under different situations. The natural variation among real life situations requires development of different types of estimators for different sampling techniques under different situations for different populations.

Systematic sampling is often advantageous over the simple random sampling for being easy to implement and often providing increased precision in estimates of population parameters of interest. Under this design, only first sampling unit is selected randomly and the subsequent units are then selected by according to certain rules. According to W.G. Madow and L.H. Madow (1944),"Systematic sampling is most commonly used probability design for the estimation of finite population parameters, due to its simplicity. "Cochran (1946) declared that apart from easy to implement, systematic sampling often provides more efficient estimators as compared to the simple random sampling (SRS) or stratified random sampling for different types of populations under different situations.

Auxiliary information is commonly used together with the main variable of interest to improve the estimates of population parameter like the mean, total and variance, etc. Ratio and regression estimation methods use auxiliary information in many ways to obtain better estimation results in terms of minimum mean square errors. Various authors have used auxiliary information to improve the estimators in terms of relative efficiency under systematic sampling. For details, readers may refer to Quenouille (1956) and Hansen et al. (1946). Swain (1964), Shukla (1971) and Singh (1967) have proposed the classical ratio, product and ratio-cumproduct-type estimators respectively under the framework of systematic sampling. Srivastava and Jhajj (1983) used multi-auxiliary variables to propose a new family of estimators. Kushwaha and Singh (1989), Banarasi et al. (1993) and Singh and Singh (1998) suggested different modified ratio, product and difference type estimators under systematic sampling. For more recent work on systematic sampling including some exponential type estimators using auxiliary information, one can refer to Singh et al. (2011), Singh and Solanki (2012), Singh and
Jatwa (2012),Tailor et al.(2013), Khan and Singh (2015) and Khan (2016).

In this paper, a generalized semi-exponential type estimator is proposed with two auxiliary variables with the expectation that this estimator will be more efficient than some of the other competing estimators considered in this paper under systematic sampling. Some existing estimators along with the methodology and useful notations of systematic sampling are given in Section 2. The expressions for approximate bias and mean square error of the proposed estimator have been derived in Section 3. Some special cases are also given in the same Section in which the proposed estimator reduces to many other exponential and nonexponential type estimators. Theoretical comparisons with some other existing estimators are addressed in Section 3. An empirical study that shows percentage relative efficiency of the proposed estimator with respect to the mean per unit estimator under systematic sampling is carried out in Section 4. Some concluding remarks are given in Section 5.

\section{Methodology of Systematic Sampling with Associated Estimators}

In this section, we introduce the following terminology that is needed in this paper. Let $y$ is the study variable and $x$ and $z$ be the auxiliary variables defined on a finite population $P$ consisting of $N$ distinct but identifiable units, $P=\left(P_{1}, P_{2}, \ldots, P_{N}\right)$, numbered in some specific order. A random sample of size $n$ is selected from the first $k$ units and then every $k^{\text {th }}$ units is selected corresponding to each unit in the sample from the first $k$ units. So there will be total $k$ samples, each of size $n$, such that $N=n k$, where $n$ and $k$ are positive integers. Let $\left(y_{i j}, x_{i j}, z_{i j}\right)$ for $i=1,2,3, \ldots, k$ and $j=1,2,3, \ldots, n$ denote the values of $j^{\text {th }}$ unit in the $i^{\text {th }}$ sample. The systematic sample means of the variable of interest and the auxiliary variables to be estimated as, $\bar{y}_{s y s}=n^{-1} \sum_{j=1}^{n} y_{i j}$, $\bar{x}_{\text {sys }}=n^{-1} \sum_{j=1}^{n} x_{i j}$ and $\bar{z}_{s y s}=n^{-1} \sum_{j=1}^{n} z_{i j}$ are unbiased estimators of the corresponding population means $\bar{Y}$, $\bar{X}$ and $\bar{Z}$ respectively.

We also denote the following error terms and the other notations: 


$$
\begin{aligned}
& \bar{y}_{s y s}=\bar{Y}\left(1+e_{y}\right) \text { and } \bar{z}_{s y s}=\bar{Z}\left(1+e_{z}\right) \\
& \text { such that } E\left(e_{y}\right)=E\left(e_{x}\right)=E\left(e_{z}\right)=0 \\
& E\left(e_{y}^{2}\right)=\theta\left(1+(n-1) \rho_{y}\right) C_{y}^{2}=C_{0}^{2}, \\
& E\left(e_{x}^{2}\right)=\theta\left(1+(n-1) \rho_{x}\right) C_{x}^{2}=C_{1}^{2} \\
& E\left(e_{z}^{2}\right)=\theta\left(1+(n-1) \rho_{z}\right) C_{z}^{2}=C_{2}^{2} \\
& E\left(e_{z}^{2}\right)=\theta\left(1+(n-1) \rho_{z}\right) C_{z}^{2}=C_{2}^{2} \\
& E\left(e_{y} e_{x}\right)=\theta\left(1+(n-1) \rho_{y}\right)^{1 / 2}\left(1+(n-1) \rho_{x}\right)^{1 / 2} \rho_{y x} C_{y} C_{x}=C_{0} C_{1} \\
& E\left(e_{y} e_{z}\right)=\theta\left(1+(n-1) \rho_{y}\right)^{1 / 2}\left(1+(n-1) \rho_{z}\right)^{1 / 2} \rho_{y z} C_{y} C_{z}=C_{0} C_{2} \\
& E\left(e_{x} e_{z}\right)=\theta\left(1+(n-1) \rho_{x}\right)^{1 / 2}\left(1+(n-1) \rho_{z}\right)^{1 / 2} \rho_{x z} C_{x} C_{z}=C_{1} C_{2} \\
& \rho_{y x}^{*}=\frac{\left(1+(n-1) \rho_{y}\right)}{\left(1+(n-1) \rho_{y}\right)}, \rho_{y z}^{*}=\frac{\left(1+(n-1) \rho_{y}\right)}{\left(1+(n-1) \rho_{z}\right)} \mathrm{and}_{x z}^{*}=\frac{\left(1+(n-1) \rho_{x}\right)}{\left(1+(n-1) \rho_{z}\right)} \\
& \rho_{y}=\frac{E\left(y_{i j}-\bar{Y}\right)\left(y_{i j}-\bar{Y}\right)}{E\left(y_{i j}-\bar{Y}\right)^{2}}, \rho_{x}=\frac{E\left(x_{i j}-\bar{X}\right)\left(x_{i j^{\prime}}-\bar{X}\right)}{E\left(x_{i j}-\bar{X}\right)^{2}} \\
& \begin{array}{c}
E\left(z_{i j}-\bar{Z}\right)\left(z_{i j}-\bar{Z}\right) \\
E\left(z_{i j}-\bar{Z}\right)^{2}
\end{array} \\
& \rho_{i j}=(N-1) / \mathrm{Nn}, \rho_{i j}^{*}=\left(1+(n-1) \rho_{i}\right) /\left(1+(n-1) \rho_{j}\right) \\
& \text { and } \rho_{z} S_{j} \text { and } H_{i j}=\rho_{i j} C_{i} / C_{j} \text { where }{ }^{2}=x, y, z \\
& j=x, y, z
\end{aligned}
$$

where $\rho_{y}, \rho_{x}$ and $\rho_{z}$ are the intraclass correlation coefficients for study variable $y$ and both auxiliary variables $x$ and $z$, respectively and $\rho_{i j}$ is the correlation coefficient between study variable and auxiliary variables. Also the quantities $C_{y}, C_{x}$ and $C_{z}$ are population coefficients of variation of the study variable and the auxiliary variables respectively.

The unbiased mean estimator, without using auxiliary information, together with the expression for the variance in systematic sampling is defined as:

$$
\begin{gathered}
t_{0}=\bar{y}_{s y s}, \\
\operatorname{var}\left(t_{0}\right)=\bar{Y}^{2} C_{0}^{2} .
\end{gathered}
$$

Swain (1964) and Shukla (1971) proposed classical ratio and product-type estimators under systematic sampling is given by

$$
\begin{aligned}
& t_{1}=\frac{\bar{y}_{\text {sys }}}{\bar{x}_{\text {sys }}} \bar{X}, \\
& t_{2}=\frac{\bar{y}_{\text {sys }}}{\bar{Z}} \bar{z}_{\text {sys }} .
\end{aligned}
$$

The expressions for the mean square error for the estimators $t_{1}$ and $t_{2}$ upto the first order of approximation are given respectively by

$$
\begin{aligned}
& \operatorname{MSE}\left(t_{1}\right)=\bar{Y}^{2}\left(C_{0}^{2}+C_{1}^{2}\left(1-2 H_{y x} \sqrt{\rho_{y x}^{*}}\right)\right), \\
& \operatorname{MSE}\left(t_{2}\right)=\bar{Y}^{2}\left(C_{0}^{2}+C_{2}^{2}\left(1+2 H_{y z} \sqrt{\rho_{y z}^{*}}\right)\right) .
\end{aligned}
$$

The traditional regression estimator for population mean under systematic sampling is given by

$$
t_{3}=\bar{y}_{s y s}+b_{y x}\left(\bar{X}-\bar{x}_{s y s}\right)
$$

where $b_{y x}$ is the sample regression coefficient between $y$ and $x$. The expression for the mean square error for the estimator $t_{3}$, up to the first order approximation is given as

$$
\operatorname{MSE}\left(t_{3}\right)=\bar{Y}^{2} C_{0}^{2}\left(1-\rho_{y x}^{2}\right) .
$$

Singh et al. (2011) proposed exponential ratio and product-type estimators for finite population mean under systematic sampling. The proposed estimators are given by

$$
\begin{aligned}
& t_{4}=\bar{y}_{s y s} \exp \left(\frac{\bar{X}-\bar{x}_{s y s}}{\bar{X}+\bar{x}_{s y s}}\right), \\
& t_{5}=\bar{y}_{s y s} \exp \left(\frac{\bar{z}_{s y s}-\bar{Z}}{\bar{Z}+\bar{z}_{s y s}}\right) .
\end{aligned}
$$

The expressions for mean square error for the estimators $t_{4}$ and $t_{5}$ using first order approximation given respectively by

$$
\begin{aligned}
& \operatorname{MSE}\left(t_{4}\right)=\bar{Y}^{2}\left(C_{0}^{2}+0.25 C_{1}^{2}\left(1-4 H_{y x} \sqrt{\rho_{y x}^{*}}\right)\right), \\
& \operatorname{MSE}\left(t_{5}\right)=\bar{Y}^{2}\left(C_{0}^{2}+0.25 C_{2}^{2}\left(1+4 H_{y z} \sqrt{\rho_{y z}^{*}}\right)\right) .
\end{aligned}
$$


Tailor et al. (2013) proposed the following ratiocum-product type estimator for finite population mean under systematic sampling:

$$
t_{6}=\bar{y}_{s y s}\left(\frac{\bar{X}}{\bar{x}_{s y s}}\right)\left(\frac{\bar{z}_{s y s}}{\bar{Z}}\right)
$$

The expression for the mean square error of estimator $t_{6}$, up to first order approximation, is given by:

$$
\operatorname{MSE}\left(t_{6}\right)=\bar{Y}^{2}\left(C_{0}^{2}+C_{1}^{2}\left(1-2 H_{y x} \sqrt{\rho_{y x}^{*}}\right)+C_{2}^{2}\left(1+2 H_{y z} \sqrt{*_{y z}^{*}}-2 H_{x z} \sqrt{\rho_{x z}^{*}}\right)\right) \cdot(15)
$$

Khan (2016) proposed a generalized class of exponential estimators for the estimation of finite population mean under systematic sampling. The estimator with the expression for the mean square error is given by:

$$
\begin{gathered}
t_{7}=\bar{y}_{s y s} \exp \left(\alpha \frac{\bar{X}-\bar{x}_{s y s}}{\bar{X}+(a-1) \bar{x}_{s y s}}+\beta \frac{\bar{Z}-\bar{z}_{s y s}}{\bar{Z}+(b-1) \bar{z}_{s y s}}\right), \\
\operatorname{MSE}\left(t_{6}\right)=\bar{Y}^{2}\left(\begin{array}{l}
C_{0}^{2}+J C_{1}^{2}\left(J-2 H_{y x} \sqrt{\rho_{y x}^{*}}\right) \\
+K C_{2}^{2}\left(K-2 H_{y z} \sqrt{\rho_{y z}^{*}}+2 J H_{x z} \sqrt{\rho_{x z}^{*}}\right)
\end{array}\right),
\end{gathered}
$$

where

$$
a=\frac{\alpha}{J}, \quad J=\sqrt{\rho_{y x}^{*}} \frac{H_{y x}-H_{y z} H_{z x}}{\left(1-\rho_{x z}^{2}\right)},
$$

and

$$
b=\frac{\beta}{M}, \quad M=\sqrt{\rho_{y z}^{*}} \frac{H_{y z}-H_{y x} H_{x z}}{\left(1-\rho_{x z}^{2}\right)} .
$$

\section{Generalized Semi-Exponential Type Estimator}

In this section, a generalized semi-exponential type mean estimator is proposed making use of two auxiliary variables under the framework of systematic sampling. The proposed estimator is:

$$
t_{G E}=\bar{y}_{s y s}\left(\frac{\bar{X}}{\bar{x}_{s y s}}\right)^{v_{1}} \exp \left(\alpha \frac{\bar{Z}-\bar{z}_{s y s}}{\bar{Z}+\left(v_{2}-1\right) \bar{z}_{s y s}}\right),
$$

where $v_{1}$ and $v_{2}\left(v_{2}>0\right)$ are constants that need to be optimized and estimated for the expression of minimum value of the mean square error of the proposed estimator $t_{G E}$. A generalized constant $\alpha$ can assume values $-1,0$ and 1 to give various special cases of the proposed estimators.

In order to obtain the expression of mean square error of the proposed estimator, we expand the proposed estimator expression using the notations given in (1), and get.

$$
t_{G E}=\bar{Y}\left(1+e_{y}\right)\left(\frac{\bar{X}}{\bar{X}\left(1+e_{x}\right)}\right)^{v_{1}} \exp \left(\alpha \frac{\bar{Z}-\bar{Z}-\bar{Z} e_{z}}{\bar{Z}+\bar{Z}\left(v_{2}-1\right)\left(1+\bar{Z} e_{z}\right)}\right) \text {. }
$$

Applying Taylor series and ignoring the terms beyond the second order of approximation and taking expectation on both sides of (19), we get

$$
\begin{aligned}
& E\left(t_{G E}-\bar{Y}\right)=\bar{Y} E\left(e_{y}-e_{x}-v_{1} e_{y} e_{x}+v_{1}^{2} e_{x}^{2}\right) \\
& \left(1-\left\{-\frac{\alpha}{v_{2}} e_{z}+\frac{\alpha}{v_{2}} e_{z}^{2}-\frac{\alpha}{v_{2}^{2}} e_{z}^{2}\right\}+\frac{\alpha^{2}}{v_{2}^{2}} e_{z}^{2}\right) .
\end{aligned}
$$

After simplification of (20), we have

$$
\operatorname{bias}\left(t_{G E}\right)=\bar{Y}\left(\begin{array}{l}
v_{1} C_{1}^{2}\left(1-H_{y x} \sqrt{\rho_{y x}^{*}}\right)+\frac{\alpha}{2 a^{2}} C_{2}^{2} \\
\left\{2(a-1)+\alpha-H_{y z} \sqrt{\rho_{y z}^{*}}-v_{1} H_{z x} \sqrt{\rho_{z x}^{*}}\right.
\end{array}\right) .
$$

In order to obtain the expression for the mean square error of the proposed estimator, applying Taylor series and ignoring the terms up to the first order approximation of (19), we have

$$
t_{G E}=\bar{Y}\left(1+e_{y}-v_{1} e_{x}\right) \exp \left(-\frac{\alpha}{v_{2}} e_{z}\right)
$$

Squaring and taking expectation on both sides of (22), we get

$$
E\left(t_{G E}-\bar{Y}\right)^{2}=\bar{Y}^{2} E\left(e_{y}-v_{1} e_{x}-\frac{\alpha}{v_{2}} e_{z}\right)^{2} .
$$

The expression for the mean square error of the proposed estimator is given as

$$
\operatorname{MSE}\left(t_{G E}\right)=\bar{Y}^{2}\left(\begin{array}{l}
C_{0}^{2}+v_{1} C_{1}^{2}\left(v_{1}-2 H_{y x} \sqrt{\rho_{y x}^{*}}\right)+\frac{\alpha}{v_{2}} C_{2}^{2} \\
\left\{\frac{\alpha}{v_{2}}-2 H_{y z} \sqrt{\rho_{y z}^{*}}+2 v_{1} H_{x z} \sqrt{\rho_{x z}^{*}}\right\}
\end{array}\right\} .
$$


The optimum values of $v_{l}$ and $v_{2}$ are

$$
v_{1}=\sqrt{\rho_{y x}^{*}} \frac{H_{y x}-H_{y z} H_{z x}}{\left(1-\rho_{x z}^{2}\right)}=J \quad \text { and } v_{2(o p t)}=\frac{\alpha}{M},
$$

where

$$
M=\sqrt{\rho_{y z}^{*}} \frac{H_{y z}-H_{y x} H_{x z}}{\left(1-\rho_{x z}^{2}\right)} .
$$

The expression of minimum mean square error of the proposed estimator $t_{G E}$ is given as:

$$
M S E_{\text {min }}\left(t_{s y s G E}\right)=\bar{Y}^{2}\left(\begin{array}{l}
C_{0}^{2}+J C_{1}^{2}\left(J-2 H_{y x} \sqrt{\rho_{y x}^{*}}\right)+M C_{2}^{2} \\
\left\{M-2 H_{y z} \sqrt{\rho_{y z}^{*}}+2 J H_{x z} \sqrt{\rho_{x z}^{*}}\right\}
\end{array}\right) .
$$

It is noticed that for different values of $v_{1}, v_{2}$ and $\alpha$ we may get various forms of exponential and semi exponential type estimators as new families of $t_{G E}$, as given in Table 1 (Appendix A).

\section{Relative Performance of Proposed Estimator Compared to other Estimators}

In this section, the theoretical comparisons of the proposed estimator are given with some relevant competing estimators.

i. The proposed generalized semi exponential type estimator $t_{G E}$ will be more precise estimator then the unbiased mean per unit estimator given in (3) when

$$
M S E_{\text {min }}\left(t_{G E}\right) \leq \operatorname{Var}\left(t_{0}\right),
$$

$$
\bar{Y}^{2}\left(\begin{array}{l}
C_{0}^{2}+J C_{1}^{2}\left(J-2 H_{y x} \sqrt{\rho_{y x}^{*}}\right)+ \\
M C_{2}^{2}\left(M-2 H_{y z} \sqrt{\rho_{y z}^{*}}+2 J H_{x z} \sqrt{\rho_{x z}^{*}}\right)
\end{array}\right) \leq \bar{Y}^{2} C_{0}^{2},
$$

$$
M C_{2}^{2}\left(2 H_{y z} \sqrt{\rho_{y z}^{*}}-M-2 J H_{x z} \sqrt{\rho_{x z}^{*}}\right)-J C_{1}^{2}\left(J-2 H_{y x} \sqrt{\rho_{y x}^{*}}\right) \geq 0 .
$$

ii. The proposed estimator $t_{G E}$ will be more precise estimator than the classical ratio estimator given in (6) when

$$
M S E_{\text {min }}\left(t_{G E}\right) \leq M S E\left(t_{1}\right),
$$

$$
\begin{aligned}
& \bar{Y}^{2}\left(\begin{array}{l}
C_{0}^{2}+J C_{1}^{2}\left(J-2 H_{y x} \sqrt{\rho_{y x}^{*}}\right)+ \\
M C_{2}^{2}\left\{M-2 H_{y z} \sqrt{\rho_{y z}^{*}}+2 J H_{x z} \sqrt{\rho_{x z}^{*}}\right\}
\end{array}\right) \\
& \leq \bar{Y}^{2}\left(C_{0}^{2}+C_{1}^{2}\left(1-2 H_{y x} \sqrt{\rho_{y x}^{*}}\right)\right)
\end{aligned}
$$

$$
\begin{aligned}
& C_{1}^{2}\left(1-2 H_{y x} \sqrt{\rho_{y x}^{*}}\right)-J C_{1}^{2}\left(J-2 H_{y x} \sqrt{\rho_{y x}^{*}}\right) \\
& -M C_{2}^{2}\left(M-2 H_{y z} \sqrt{\rho_{y z}^{*}}+2 J H_{x z} \sqrt{\rho_{x z}^{*}}\right) \geq 0 .
\end{aligned}
$$

iii. The proposed estimator $t_{G E}$ will be more precise estimator than the product estimator given in (7) when

$$
\begin{gathered}
M S E_{\text {min }}\left(t_{G E}\right) \leq \operatorname{MSE}\left(t_{2}\right) . \\
A C_{1}^{2}\left(A-2 H_{y x} \sqrt{\rho_{y x}^{*}}\right)+M C_{2}^{2}\left(M-2 H_{y z} \sqrt{\rho_{y z}^{*}}+2 A H_{x z} \sqrt{\rho_{x z}^{*}}\right) \\
\leq C_{2}^{2}\left(1+2 H_{y z} \sqrt{\rho_{y z}^{*}}\right), \\
C_{2}^{2}\left(1+2 H_{y z} \sqrt{\rho_{y z}^{*}}\right)-A C_{1}^{2}\left(A-2 H_{y x} \sqrt{\rho_{y x}^{*}}\right) \\
-M C_{2}^{2}\left(M-2 H_{y z} \sqrt{\rho_{y z}^{*}}+2 A H_{x z} \sqrt{\rho_{x z}^{*}}\right) \geq 0 .
\end{gathered}
$$

iv. The proposed estimator $t_{G E}$ will be more precise estimator than the regression estimator given in (9) when

$$
\begin{gathered}
M S E_{\text {min }}\left(t_{G E}\right) \leq M S E\left(t_{3}\right), \\
\bar{Y}^{2}\left(\begin{array}{l}
C_{0}^{2}+J C_{1}^{2}\left(J-2 H_{y x} \sqrt{\rho_{y x}^{*}}\right)+M C_{2}^{2} \\
\left(M-2 H_{y z} \sqrt{\rho_{y z}^{*}}+2 J H_{x z} \sqrt{\rho_{x z}^{*}}\right)
\end{array}\right) \leq \bar{Y}^{2} C_{0}^{2}\left(1-\rho_{y x}^{2}\right), \\
\rho_{y x}^{2} \geq\left(\begin{array}{l}
J C_{1}^{2}\left(2 H_{y x} \sqrt{\rho_{y x}^{*}}-J\right)+M C_{2}^{2} \\
\left(2 H_{y z} \sqrt{\rho_{y z}^{*}}-M-2 J H_{x z} \sqrt{\rho_{x z}^{*}}\right)
\end{array}\right) / C_{0}^{2} .
\end{gathered}
$$

v. The proposed estimator $t_{G E}$ will be more precise estimator than the exponential ratio type estimator given in (12) when

$$
\begin{gathered}
\operatorname{MSE}_{\text {min }}\left(t_{G E}\right) \leq \operatorname{MSE}\left(t_{4}\right), \\
\bar{Y}^{2}\left(C_{0}^{2}+J C_{1}^{2}\left(J-2 H_{y x} \sqrt{\rho_{y x}^{*}}\right)+M C_{2}^{2}\left(M-2 H_{y z} \sqrt{\rho_{y z}^{*}}+2 J H_{x z} \sqrt{\rho_{x z}^{*}}\right)\right) \\
\leq \bar{Y}^{2}\left(C_{0}^{2}+0.25 C_{1}^{2}\left(1-4 H_{y x} \sqrt{\rho_{y x}^{*}}\right)\right),
\end{gathered}
$$




$$
\begin{aligned}
& C_{1}^{2}\left(0.25-4 H_{y x} \sqrt{\rho_{y x}^{*}}\right)-J C_{1}^{2}\left(J-2 H_{y x} \sqrt{\rho_{y x}^{*}}\right) \\
& -M C_{2}^{2}\left(M-2 H_{y z} \sqrt{\rho_{y z}^{*}}+2 J H_{x z} \sqrt{\rho_{x z}^{*}}\right) \geq 0 .
\end{aligned}
$$

vi. The proposed estimator $t_{G E}$ will be more precise estimator than the exponential product-type estimator given in (13) when

$$
\begin{gathered}
M S E_{\min }\left(t_{G E}\right) \leq \operatorname{MSE}\left(t_{5}\right), \\
\bar{Y}^{2}\left(C_{0}^{2}+J C_{1}^{2}\left(J-2 H_{y x} \sqrt{\rho_{y x}^{*}}\right)+M C_{2}^{2}\left(M-2 H_{y z} \sqrt{\rho_{y z}^{*}}+2 J H_{x z} \sqrt{\rho_{x z}^{*}}\right)\right) \\
\leq \bar{Y}^{2}\left(C_{0}^{2}+0.25 C_{2}^{2}\left(1+4 H_{y z} \sqrt{\rho_{y z}^{*}}\right)\right), \\
C_{2}^{2}\left(0.25+4 H_{y z} \sqrt{\rho_{y z}^{*}}\right)-J C_{1}^{2}\left(J-2 H_{y x} \sqrt{\rho_{y x}^{*}}\right) \\
-M C_{2}^{2}\left(M-2 H_{y z} \sqrt{\rho_{y z}^{*}}+2 J H_{x z} \sqrt{\rho_{x z}^{*}}\right) \geq 0 .
\end{gathered}
$$

vii. The proposed estimator $t_{G E}$ will be more precise estimator than the ratio-cum-product type estimator given in (15) when

$$
\begin{gathered}
M S E_{\min }\left(t_{G E}\right) \leq \operatorname{MSE}\left(t_{6}\right), \\
\bar{Y}^{2}\left(C_{0}^{2}+J C_{1}^{2}\left(J-2 H_{y x} \sqrt{\rho_{y x}^{*}}\right)+M C_{2}^{2}\left(M-2 H_{y z} \sqrt{\rho_{y z}^{*}}+2 J H_{x z} \sqrt{\rho_{x z}^{*}}\right)\right) \\
\leq \bar{Y}^{2}\left(C_{0}^{2}+C_{1}^{2}\left(1-2 H_{y x} \sqrt{\rho_{y x}^{*}}\right)+C_{2}^{2}\left(1+2 H_{y z} \sqrt{\rho_{y z}^{*}}-2 H_{x z} \sqrt{\rho_{x z}^{*}}\right)\right), \\
C_{1}^{2}\left(1-2 H_{y x} \sqrt{\rho_{y x}^{*}}\right)+C_{2}^{2}\left(1+2 H_{y z} \sqrt{\rho_{y z}^{*}}-2 H_{x z} \sqrt{\rho_{x z}^{*}}\right)-J C_{1}^{2} \\
\left(J-2 H_{y x} \sqrt{\rho_{y x}^{*}}\right)-M C_{2}^{2}\left(M-2 H_{y z} \sqrt{\rho_{y z}^{*}}+2 J H_{x z} \sqrt{\rho_{x z}^{*}}\right) \geq 0 .
\end{gathered}
$$

viii. The proposed estimator $t_{G E}$ will be more precise estimator than the generalized exponential-cum-exponential type estimator given in (17) when

$$
\begin{gathered}
\operatorname{MSE}_{\min }\left(t_{G E}\right) \leq \operatorname{MSE}\left(t_{7}\right), \\
\bar{Y}^{2}\left(C_{0}^{2}+J C_{1}^{2}\left(J-2 H_{y x} \sqrt{\rho_{y x}^{*}}\right)+M C_{2}^{2}\left(M-2 H_{y z} \sqrt{\rho_{y z}^{*}}+2 J H_{x z} \sqrt{\rho_{x z}^{*}}\right)\right) \\
\leq \bar{Y}^{2}\left(C_{0}^{2}+J C_{1}^{2}\left(J-2 H_{y x} \sqrt{\rho_{y x}^{*}}\right)+K C_{2}^{2}\left(K-2 H_{y z} \sqrt{\rho_{y z}^{*}}+2 J H_{x z} \sqrt{\rho_{x z}^{*}}\right)\right), \\
C_{1}^{2}\left(J-H_{y x} \sqrt{\rho_{y x}^{*}}\right)+M C_{2}^{2} H_{x z} \sqrt{\rho_{x z}^{*}} \geq 0 .
\end{gathered}
$$

\section{Empirical Study}

Evaluation of the proposed estimator is based on the percentage relative efficiencies (PRE's) compared to the traditional unbiased mean estimator. So the value greater than one hundred indicates that the competing estimators are more efficient than the usual mean estimator. The PRE's of all the estimators over the mean per unit estimator can be obtained from the following mathematical formula:

$$
\operatorname{PRE}=\frac{\operatorname{var}\left(t_{0}\right)}{\operatorname{MSE}\left(t_{i}\right)} \times 100
$$

$$
i=0,1,2, \ldots, 7, G 1, G 2, \ldots, G 10 .
$$

Here, we consider a population data set from the literature given in Table 2, to examine the performance of the proposed estimator over the other competing estimators at optimum conditions.

\section{Table2: Population (Source: Tailor et al. (2013)}

\begin{tabular}{llllll}
\hline$N$ & 15 & $n$ & \multicolumn{1}{c}{3} & - & - \\
$\bar{Y}$ & 80 & $C_{y}$ & 0.56 & $\rho_{y x}$ & 0.9848 \\
$\bar{X}$ & 44.47 & $C_{x}$ & 0.28 & $\rho_{y z}$ & -0.9760 \\
$\bar{Z}$ & 48.40 & $C_{z}$ & 0.43 & $\rho_{x z}$ & -0.9539 \\
$S_{y x}$ & 538.57 & $S_{y}^{2}$ & 2000 & $\rho_{y}$ & 0.6652 \\
$S_{y z}$ & -902.87 & $S_{x}^{2}$ & 149.55 & $\rho_{x}$ & 0.7070 \\
$S_{x z}$ & -241.06 & $S_{z}^{2}$ & 427.83 & $\rho_{z}$ & 0.5487 \\
\hline
\end{tabular}

The results of MSE's and PRE's of all the estimators considered in this paper are summarized in Table 3.

Table 3: MSE's and PRE's of all the estimators

\begin{tabular}{llllll}
\hline$t_{\boldsymbol{i}}$ & $\boldsymbol{M S E}$ & $\boldsymbol{P R E} \boldsymbol{\prime} \boldsymbol{s}$ & $t_{i}$ & $\boldsymbol{M S E}$ & $\boldsymbol{P R E}$ 's \\
\hline$t_{0}$ & 1344.07 & 100.00 & $t_{G 1}$ & 4408.34 & 33.01 \\
$t_{1}$ & 373.32 & 389.62 & $t_{G 2}$ & 46.27 & 3144.94 \\
$t_{2}$ & 768.06 & 189.45 & $t_{G 3}$ & 2249.14 & 64.70 \\
$t_{3}$ & 43.74 & 3362.67 & $t_{G 4}$ & 1131.00 & 128.66 \\
$t_{4}$ & 820.09 & 177.43 & $t_{G 5}$ & 93.11 & 1562.88 \\
$t_{5}$ & 1044.42 & 139.32 & $t_{G 6}$ & 52.99 & 2746.27 \\
$t_{6}$ & 187.08 & 777.79 & $t_{G 7}$ & 7321.20 & 19.88 \\
$t_{7}$ & 23.67 & 6158.08 & $t_{78}$ & 5100.04 & 28.53 \\
$\boldsymbol{t}_{\boldsymbol{G E}}$ & $\mathbf{2 1 . 6 2}$ & $\mathbf{6 7 2 9 . 2 1}$ & $t_{G 9}$ & 753.10 & 193.22 \\
& & & $t_{G 10}$ & 1816.00 & 80.13 \\
\hline
\end{tabular}

The results presented in Table 3 indicate that the proposed generalized semi-exponential type estimator works considerably better than all other exponential and non-exponential estimators considered in this paper. The proposed estimator has the least mean square error as compare to other estimators.

\section{Conclusion}

A generalized semi-exponential type estimator is proposed in this paper for the estimation of finite 
population mean with two auxiliary variables under the framework of systematic sampling. The expressions of approximate bias and mean square error of the proposed estimator are derived. The algebraic expressions for the mean square error of the proposed estimator are compared with other existing estimators both theoretically and empirically. The efficiency comparisons are also carried out using the data taken from Tailor et al. (2013). The results illustrated in Table 3 show that the proposed generalized semi-exponential type estimator is more efficient than the other estimators considered in this paper in term of higher percent relative efficiency.

\section{Acknowledgement}

Authors are thankful to the editor-in-chief and the learned referee for their inspiring and fruitful suggestions

\section{References}

1. A.K.P.C. Swain, "The use of systematic sampling ratio estimate," Journal of the Indian Statistical Association, 2 (1964) 160-164

2. Banarasi, S. N. S. Kushwaha, and K. S. Kushwaha, A class of ratio, product and difference type estimators in systematic sampling, Microelectronics Reliability. 33(4) (1993) 455- 457

3. H. P. Singh and R. Singh, Almost unbiased ratio and product type estimators in systematic sampling, Questiio, 22(3) (1998) 403-416

4. H. P. Singh, R. Tailor, and N. K. Jatwa, Modified ratio and product estimators for population mean in systematic sampling, Journal of Modern Applied Statistical Methods, 10(2), (2011) 424435

5. H. P. Singh and R. S. Solanki, An efficient class of estimators for the population mean using auxiliary information in systematic sampling, Journal of Statistical Theory and Practice, 6 (2), (2012) 274-285
6. H. P. Singh and N. K. Jatwa, A class of exponential type estimators in systematic sampling, Economic, Quality Control, 27(2), (2012) 195-208

7. K. S. Kushwaha and H. P. Singh, Class of almost unbiased ratio and product estimators in systematic sampling, Journal of theIndian Society of Agricultural Statistics,. 41(2), (1989) 193- 205

8. M. H. Quenouille, Notes on bias in estimation, Biometrika, 43, (1956) 353-360

9. M. H. Hansen, W. N. Hurwitz, and M. Gurney, Problems and methods of the sample survey of business, Journal of the American Statistical Association, 41(234) (1946) 173-189

10. M. Khan and R. Singh, Estimation of population mean in chain ratio-type estimator under systematic sampling, Journal of Probability and Statistics, (2015), Article ID248374, 2 pages.

11. M. Khan, A general class of exponential type estimator for population mean under systematic sampling using two auxiliary variables, Journal of Probability and Statistics, (2015), Article ID2374837, 6 pages.

12. M. P. Singh, Ratio-cum-product method of estimation, Metrika, 12 (1967) 34-42

13. N. D. Shukla, Systematic sampling and product method of estimation," in Proceeding of the all India Seminar on Demography and Statistics, (Varanasi, India 1971)

14. S. K. Srivastava and H. S. Jhajj, A class of estimators of the population mean using multiauxiliary information, Calcutta Statistical Association Bulletin, 32, (1983) 47-56

15. T. Tailor, N. Jatwa, and H. P. Singh, A ratio-cumproduct estimator of finite population mean in systematic sampling, Statistics in Transition, 14(3) (2013) 391-398

16. W. G. Madow and L. H. Madow, On the theory of systematic sampling, Annals of Mathematical Statistics, 15 (1944) 1-24

17. W. G. Cochran, Relative accuracy of systematic and stratified random samples for a certain class of populations, The Annals of Mathematical Statistics, 17 (1946) 164-177 


\section{APPENDIX A}

Table 1: Some Special Cases of Proposed Estimator

\begin{tabular}{|c|c|c|c|c|}
\hline Estimators & Mean Square Errors & $v_{1}$ & $\alpha$ & $v_{2}$ \\
\hline$t_{0}=\bar{y}_{s y s}$ & $\operatorname{MSE}\left(t_{0}\right)=\bar{Y}^{2} C_{0}^{2}$ & 0 & 0 & 0 \\
\hline$t_{G 1}=\bar{y}_{s y s} \exp \left(\frac{\bar{Z}-\bar{z}_{s y s}}{\bar{Z}}\right)$ & $\operatorname{MSE}\left(t_{G 1}\right)=\bar{Y}^{2}\left(C_{0}^{2}+C_{2}^{2}\left(1-2 H_{y z} \sqrt{\rho_{y z}^{*}}\right)\right)$ & 0 & 1 & 1 \\
\hline$t_{4}=\bar{y}_{s y s} \exp \left(\frac{\bar{Z}-\bar{z}_{s y s}}{\bar{Z}+\bar{z}_{s y s}}\right)$ & $\operatorname{MSE}\left(t_{4}\right)=\bar{Y}^{2}\left(C_{0}^{2}+0.25 C_{2}^{2}\left(1-4 H_{y z} \sqrt{\rho_{y z}^{*}}\right)\right)$ & 0 & 1 & 2 \\
\hline$t_{G 2}=\bar{y}_{s y s} \exp \left(\frac{\bar{z}_{\text {sys }}-\bar{Z}}{\bar{Z}}\right)$ & $\operatorname{MSE}\left(t_{G 2}\right)=\bar{Y}^{2}\left(C_{0}^{2}+C_{2}^{2}(1\right.$ & 0 & -1 & 1 \\
\hline$t_{5}=\bar{y}_{s y s} \exp \left(\frac{\bar{z}_{s y s}-\bar{Z}}{\bar{Z}+\bar{z}_{s y s}}\right)$ & $\operatorname{MSE}\left(t_{5}\right)=\bar{Y}^{2}\left(C_{0}^{2}+0.25 C_{2}^{2}(1\right.$ & 0 & -1 & 2 \\
\hline$t_{1}=\bar{y}_{s y s}\left(\frac{\bar{X}}{\bar{x}_{s y s}}\right)$ & $\operatorname{MSE}\left(t_{1}\right)=\bar{Y}^{2}\left(C_{0}^{2}+C_{1}^{2}(1-2 H\right.$ & 1 & 0 & 0 \\
\hline$t_{G 3}=\bar{y}_{s y s}\left(\frac{\bar{X}}{\bar{x}_{s y s}}\right) \exp \left(\frac{\bar{Z}-\bar{z}_{s y s}}{\bar{Z}}\right)$ & $\left.\left.\begin{array}{c}* \\
{ }_{y x}\end{array}\right)+C_{2}^{2}\left(1-2 H_{y z} \sqrt{\rho_{y z}^{*}}+2 H_{x z} \sqrt{\rho_{x z}^{*}}\right)\right)$ & 1 & 1 & 1 \\
\hline$t_{G 4}=\bar{y}_{s y s}\left(\frac{\bar{X}}{\bar{x}_{s y s}}\right) \exp \left(\frac{\bar{Z}-\bar{z}_{\text {sys }}}{\bar{Z}+\bar{z}_{s y s}}\right)$ & $\operatorname{MSE}\left(t_{G 4}\right)=\bar{Y}^{2}\left(C_{0}^{2}+C_{1}^{2}\left(1-2 H_{y x} \sqrt{\rho_{y x}^{*}}\right)+0.25 C_{2}^{2}\left(1-4 H_{y z} \sqrt{\rho_{y z}^{*}}+4 H_{x z} \sqrt{\rho_{x z}^{*}}\right)\right)$ & 1 & 1 & 2 \\
\hline$t_{G 5}=\bar{y}_{s y s}\left(\frac{\bar{X}}{\bar{x}_{s y s}}\right) \exp \left(\frac{\bar{z}_{s y s}-\bar{Z}}{\bar{Z}}\right)$ & $\operatorname{MSE}\left(t_{G 5}\right)=\bar{Y}^{2}\left(C_{0}^{2}+C_{1}^{2}\left(1-2 H_{y}\right)\right.$ & 1 & -1 & 1 \\
\hline$t_{G 6}=\bar{y}_{s y s}\left(\frac{\bar{X}}{\bar{x}_{s y s}}\right) \exp \left(\frac{\bar{z}_{s y s}-\bar{Z}}{\bar{Z}+\bar{z}_{s y s}}\right)$ & )$\left.+0.25 C_{2}^{2}\left(1+4 H_{y z} \sqrt{\rho_{y z}^{*}}-4 H_{x z} \sqrt{\rho_{x z}^{*}}\right)\right)$ & 1 & -1 & 2 \\
\hline$t_{2}=\bar{y}_{s y s}\left(\frac{\bar{x}_{s y s}}{\bar{X}}\right)$ & $\operatorname{MSE}\left(t_{1}\right)=\bar{Y}^{2}\left(C_{0}^{2}+C_{1}^{2}\left(1+2 H_{y x}\right.\right.$ & -1 & 0 & 0 \\
\hline$t_{G 7}=\bar{y}_{s y s}\left(\frac{\bar{x}_{s y s}}{\bar{X}}\right) \exp \left(\frac{\bar{Z}-\bar{z}_{s y s}}{\bar{Z}}\right)$ & $\operatorname{MSE}\left(t_{G 7}\right)=\bar{Y}^{2}\left(C_{0}^{2}+C_{1}^{2}\left(1+2 H_{y x} \sqrt{\rho_{y x}^{*}}\right)+C_{2}^{2}\left(1-2 H_{y z} \sqrt{\rho_{y z}^{*}}-2 H_{x z} \sqrt{\rho_{x z}^{*}}\right)\right)$ & -1 & 1 & 1 \\
\hline$t_{G 8}=\bar{y}_{s y s}\left(\frac{\bar{x}_{s y s}}{\bar{X}}\right) \exp \left(\frac{\bar{Z}-\bar{z}_{s y s}}{\bar{Z}+\bar{z}_{s y s}}\right)$ & $\operatorname{MSE}\left(t_{G 8}\right)=\bar{Y}^{2}\left(C_{0}^{2}+C_{1}^{2}\left(1+2 H_{y x} \sqrt{\rho_{y x}^{*}}\right)+0.25 C_{2}^{2}\left(1-4 H_{y z} \sqrt{\rho_{y z}^{*}}-4 H_{x z} \sqrt{\rho_{x z}^{*}}\right)\right)$ & -1 & 1 & 2 \\
\hline$t_{G 9}=\bar{y}_{s y s}\left(\frac{\bar{x}_{s y s}}{\bar{X}}\right) \exp \left(\frac{\bar{z}_{s y s}-\bar{Z}}{\bar{Z}}\right)$ & $\operatorname{MSE}\left(t_{G 9}\right)=\bar{Y}^{2}\left(C_{0}^{2}+C_{1}^{2}\left(1+2 H_{y x} \sqrt{\rho_{y x}^{*}}\right)+C_{2}^{2}\left(1+2 H_{y z} \sqrt{\rho_{y z}^{*}}+2 H_{x z} \sqrt{\rho_{x z}^{*}}\right)\right)$ & -1 & -1 & 1 \\
\hline$t_{G 10}=\bar{y}_{s y s}\left(\frac{\bar{x}_{s y s}}{\bar{X}}\right) \exp \left(\frac{\bar{z}_{s y s}-\bar{Z}}{\bar{Z}+\bar{z}_{s y s}}\right)$ & $\operatorname{MSE}\left(t_{G 10}\right)=\bar{Y}^{2}\left(C_{0}^{2}+C_{1}^{2}\left(1+2 H_{y x} \sqrt{\rho_{y x}^{*}}\right)+0.25 C_{2}^{2}\left(1+4 H_{y z} \sqrt{\rho_{y z}^{*}}+4 H_{x z} \sqrt{\rho_{x z}^{*}}\right)\right)$ & -1 & -1 & 2 \\
\hline
\end{tabular}

